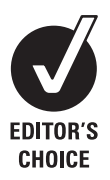
Department of Pathology,
University Health Network University of Toronto, Toronto, Canada

Correspondence to: Professor R Chetty, Department of Pathology, University Health Network, The Toronto General Hospital, 200 Elizabeth Street, 11 th Floor, Eaton Wing, Toronto, Ontario, M5G 2C4, Canada; runjan.chetty@uhn.on.ca

Accepted 16 July 2008

\title{
Revision 2: an immunohistochemical approach and evaluation of solid pseudopapillary tumour of the pancreas
}

\author{
S Serra, R Chetty
}

\begin{abstract}
Solid pseudopapillary tumours (SPT) of the pancreas are uncommon, but with widespread and increased imaging, several of these lesions are coming to light incidentally and are subject to needle biopsies. On limited material and especially the solid or clear cell, variants of SPT can morphologically mimic most notably pancreatic neuroendocrine tumours and even metastatic renal cell carcinoma or melanoma. In this context, immunohistochemistry is important and useful in helping to reach the correct diagnosis. Several antibodies have been used in the immunohistochemical evaluation of SPT. As with most tumours, no one marker is specific, but rather a core panel is advocated. Recently, both $\beta$-catenin and Ecadherin have been shown to be of value in SPT. Nuclear and cytoplasmic decoration of tumour cells by $\beta$-catenin is seen in almost $100 \%$ of cases. This protein relocalisation away from the cell membrane is underscored by mutations of the $\beta$-catenin gene. Mutations of the $\mathrm{CDH1}$ gene are very uncommon in SPT, but the immunohistochemically detected changes to the protein are consistent and present in $100 \%$ of cases. Using an Ecadherin antibody to the extracellular domain of the molecule results in complete membrane loss, while the antibody directed to the cytoplasmic fragment produces distinct nuclear staining of the tumour cells. In addition, there is concordance of staining abnormalities between the two antibodies. When combined with CD10 and progesterone receptor positivity, a diagnosis of SPT can be rendered with confidence even in small biopsy samples.
\end{abstract}

With the advent of sophisticated, high-resolution non-invasive imaging techniques, the detection of incidental small lesions throughout the body is increasing. Imaging of the pancreas has kept pace with this growing trend, resulting in smaller lesions being detected and investigated. So-called incidental pancreatic cystic lesions are being increasingly documented, and a recent publication quotes an incidence of $0.2-0.7 \% .{ }^{1}$ Although techniques such as multidetector computed tomography and magnetic resonance pancreatography allow for detailed visualisation and characterisation of pancreatic cysts, pathological and/or cytological evaluation remains the gold standard when it comes to reaching a definitive diagnosis. In concert with this, more image-directed needle biopsies are being sent for pathological evaluation. Inherent in this are the pitfalls of sample size and representativity, two factors that add to diagnostic difficulty even in the best of circumstances. With limited material the pathologist tends to be more dependent on ancillary techniques to confirm a morphological opinion, and immunohistochemistry is still the bedrock, routine investigation that is resorted to. Besides pancreatic pseudocysts, other intrinsic cystic lesions of the pancreas that are important are: serous cystic neoplasms, mucinous cystic neoplasms, intraductal papillary mucinous neoplasm and solid pseudopapillary tumours (SPT). ${ }^{2}$

SPT is an uncommon primary pancreatic tumour, constituting 1-2\% of all exocrine tumours of the pancreas and about $5 \%$ of cystic pancreatic tumours. ${ }^{3}$ Although its origin is still conjectural and slightly controversial, the weight of evidence points towards ductal origin. ${ }^{3}$ In general, it is a low-grade malignant tumour associated with a good prognosis after surgical excision even though widespread liver metastases and vascular invasion have been reported. ${ }^{4}$ Invasion into surrounding organs such as the spleen and duodenum is rare. ${ }^{3}$

Histologically, it is quite characteristic, if not diagnostic, in most resected cases. However, there are variants such as the predominantly solid example and even a clear cell variant that may cause mimicry of other tumours. ${ }^{5}$ The histological mimicry is heightened in needle core samples.

Recently, a few new markers that are important in the immunohistochemical work-up of SPT have emerged in the literature. The purpose of this overview is to highlight briefly the histological differential diagnosis of SPT, enumerate the various immunohistochemical markers that have been used traditionally in the work-up of SPT, discuss the more recent markers that are of diagnostic value in the identification of SPT and, finally, to provide a rational approach to the immunohistochemical work-up and diagnosis of these tumours.

\section{HISTOLOGICAL DIFFERENTIAL DIAGNOSIS}

As mentioned already, in the majority of cases, SPT is readily identifiable, and immunohistochemistry serves merely as a confirmatory tool. However, in limited material, pancreatic neuroendocrine tumours (which can also show pseudopapillary areas), acinar cell carcinoma, metastatic clear cell renal carcinoma, metastatic melanoma and even pancreaticoblastoma may display histological overlap with SPT, especially the more solid examples (fig 1) and it can be difficult to separate them on morphological grounds alone.

IMMUNOHISTOCHEMICAL MARKERS USED IN SPT A wide variety of markers have been employed in the investigation of SPT. For the sake of completeness, 


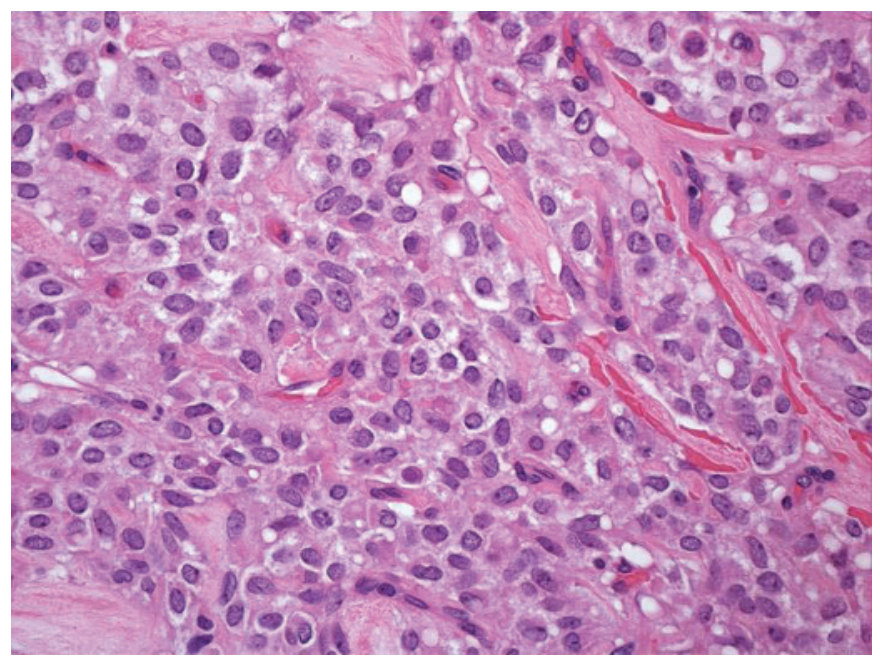

Figure 1 Predominantly solid pseudopapillary tumour with tumour cells in a nested pattern. There is a superficial resemblance to an endocrine tumour that can also have cytoplasmic vacuolisation and hyaline globules.

we will mention also that enzyme histochemistry for trypsin, $\alpha-1$ anti-trypsin, chymotrypsin, amylase and lipase have also been used to stain SPT. These enzymes have given inconsistent results and may be either positive or negative in SPT. The most consistent positivity is seen with $\alpha$-1-anti-trypsin. Trypsin and chymotrypsin are positive in approximately $20 \%$ of cases of SPT, exhibiting sparsely granular staining in the cytoplasm with a focal localisation.

In addition, they do not aid in separating SPT from acinar cell carcinoma, which is also positive for trypsin, $\alpha$-1-anti-trypsin and chymotrypsin. These histochemical stains are often messy and difficult to interpret.

\section{CD10}

CD10 or neutral endopeptidase 24.11 or neprilysin is involved in the catabolism of biological modulators and is ubiquitously distributed in various tissues. ${ }^{6}$ In both human adult and fetal pancreas, CD10 has been reported to be positive in the luminal aspect of ducts and acini.

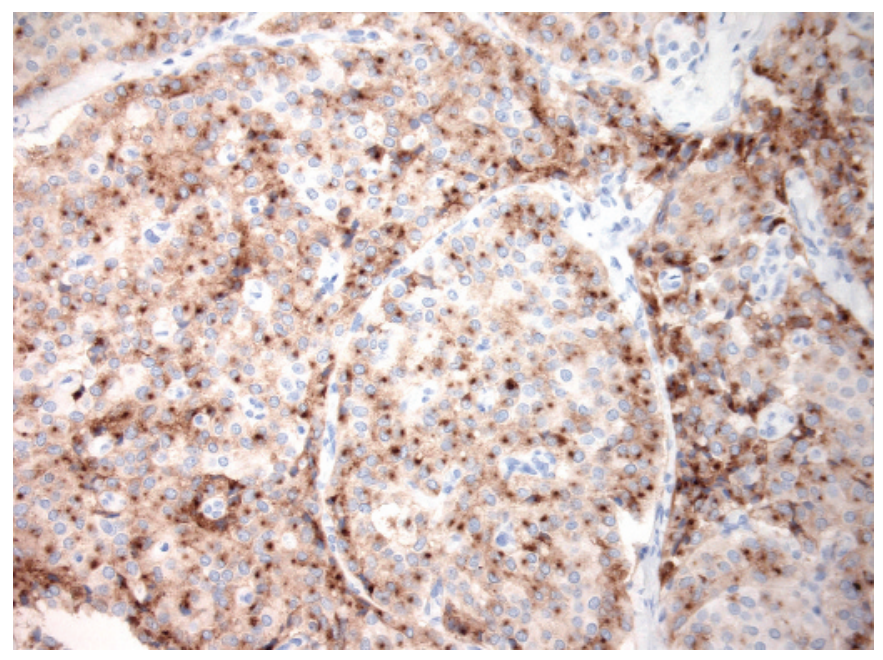

Figure 2 Typical staining pattern seen with CD10. There is cytoplasmic staining together with very characteristic dot-like accentuation.

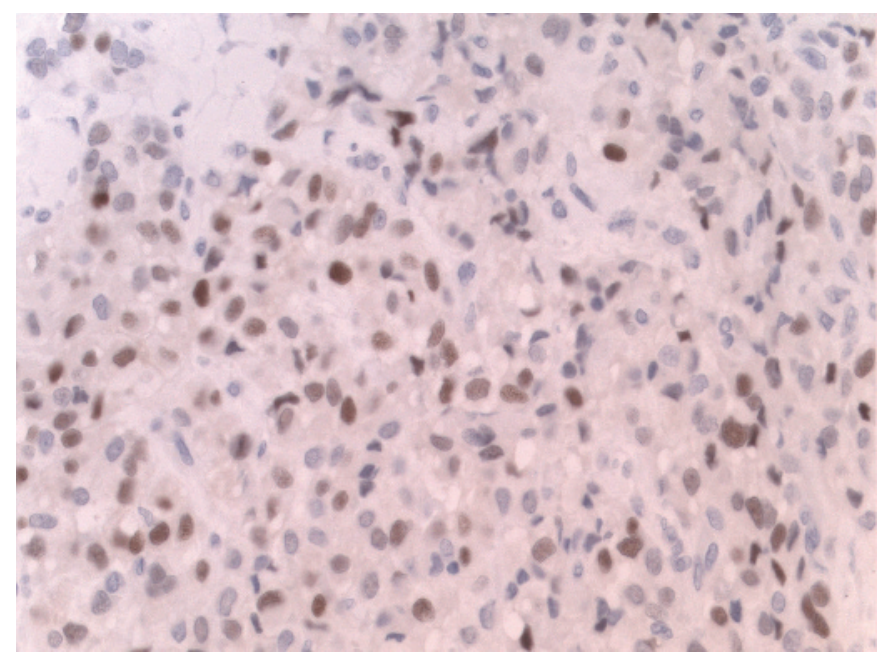

Figure 3 Progesterone receptor positivity seen in the vast majority of cases of solid pseudopapillary tumour. Staining is not diffusely distributed among all tumour cells.

CD10 immunoexpression is a consistent feature of SPT (up to $80 \%$ of cases are positive) and is usually positive in the majority of the tumour cells, is mainly localized to the cytoplasm, occasionally showing a dot-like accentuation pattern, and occasionally highlights the cell membrane (fig 2). Dot-like staining merely reflects Golgi accentuation of cytoplasmic immunolabelling. There does not appear to be any significance associated with this pattern of staining. Acinar cell carcinoma also stains with CD10 but has a luminal surface pattern of staining. Interestingly, CD10 also stains hyaline globules present in the cytoplasm of tumour cells. ${ }^{6}$ It is thought that reduced CD10 expression may promote cell proliferation as result of an increase of biological modulators, and it has been speculated that reduced/absent CD10 expression in SPT may be a predictor of an adverse clinical. CD10 has also been reported to be focally expressed in the clear cell variant of SPT. ${ }^{5}$

However, CD10 stains approximately $25 \%$ of pancreatic neuroendocrine tumours, a variety of carcinomas, including acinar cell and renal cell carcinomas, and sarcomas. Thus, CD10 is of limited value in small biopsies.

\section{Progesterone and oestrogen receptors}

Although there is a distinct female preponderance for SPT, oestrogen receptor- $\alpha$ positivity is very uncommon, and most series report absence of immunoreactivity. ${ }^{8}$ On the other hand, progesterone receptor positivity is seen in almost all cases of SPT, irrespective of gender (fig 3). Progesterone receptor immunopositivity is seen in the normal pancreatic islets, and approximately $60 \%$ of pancreatic neuroendocrine tumours show positivity for this receptor." The rationale for progesterone rather than oestrogen receptor activation is not clear but perhaps is linked to wnt pathway abnormalities in particular, nuclear $\beta$-catenin. This, in turn leads to target gene abnormalities, especially a cluster of chromosome 11q molecules that are upregulated. These include progesterone receptor, cyclin D1, FLI-1 and CD56.

\section{Neuroendocrine markers}

\section{Synaptophysin}

Synaptophysin is positive in $70 \%$ of cases: more frequently staining individual isolated cells or small clusters of cells rather 


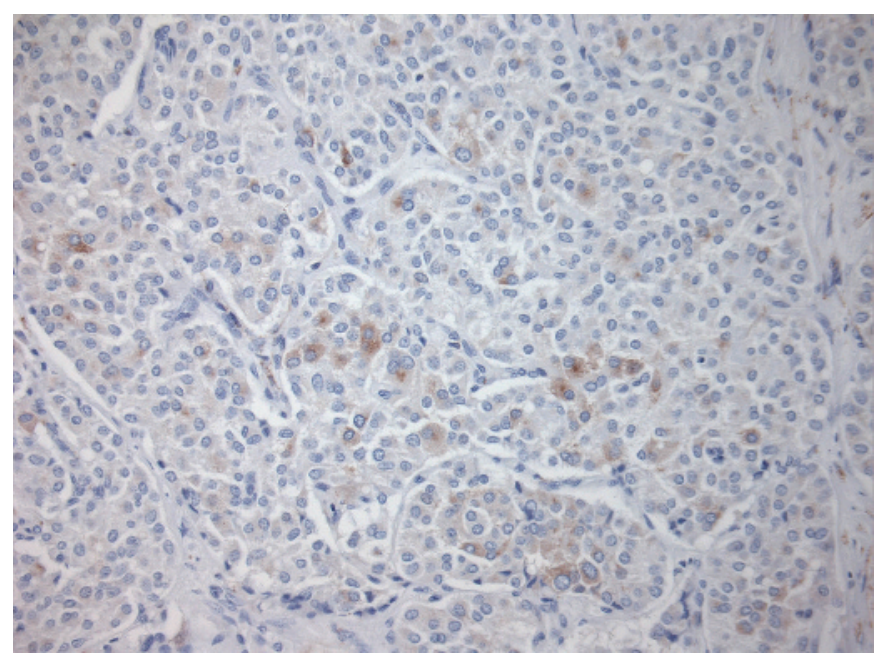

Figure 4 Synaptophysin positivity. Although commonly encountered in solid pseudopapillary tumour, it often has a cytoplasmic "blush" in contrast to strong, crisp immunoreactivity of neuroendocrine tumours.

than showing a diffuse staining pattern which is expected in neuroendocrine tumours (fig 4). If diffuse staining is encountered, then the positive staining is sparsely distributed throughout the tumour. ${ }^{6}$ Focal synaptophysin immunoexpression is the rule with SPT.

\section{Chromogranin A}

Chromogranin A is negative in all cases of SPT.

\section{CD56}

CD56 is a neural cell adhesion molecule and member of the immunoglobulin superfamily and is implicated in cell-cell or cell-matrix adhesion during development. It is expressed in both normal and neoplastic cells.
In the human pancreas, CD56 is usually expressed diffusely but weakly in adult islets of Langerhans. With regards to CD56 staining in SPT, it has been reported to be positive in $55.5-100 \%$ of cases with variable intensity. ${ }^{67}$ Not only are the predominant epithelioid cells of SPT positive, but multinucleated, foamy tumour cells as well as clear cells also stain for CD56. Foamy macrophages (not foamy tumour cells) are negative for CD56 but positive for CD68. CD56 is also positive in malignant SPT, both in the primary tumour and in the metastasis. ${ }^{6}$ Although CD56 immunoexpression has been reported to be significantly higher in metastatic cases compared with those localised to the pancreas, suggesting that it may serve as a marker for SPT with metastatic potential, a larger number of cases need to be examined to validate this hypothesis. ${ }^{7}$

CD56 also displays intense immunoreactivity in many neuroendocrine tumours and thus cannot be relied on to separate SPT from neuroendocrine tumours.

\section{Neuron-specific enolase}

Neuron-specific enolase is diffusely positive for most cases of SPT. However, it also stains $90 \%$ of melanomas, $100 \%$ of renal cell carcinomas and pancreatic neuroendocrine tumours, and $50-60 \%$ of acinar cell carcinomas. ${ }^{6} 0^{11}$ In view of this low specificity, NSE has very little use in the work-up of SPT.

Protein gene product 9.5

SPT shows only scattered positive cells in approximately $40 \%$ of cases.

\section{Cytokeratins}

AE1/AE3 is positive in up to $75 \%$ of the cases, but staining is of a weaker intensity in SPT compared with normal pancreatic epithelium. Sometimes there is an accentuation of staining in the basal portion of the tumour cells. However, results vary with different series, and it is best to regard cytokeratin expression in SPT as inconsistent and unreliable for diagnostic purposes.
Figure 5 (A) $\beta$-Catenin, the key mediator of the Wnt signal. In cells not exposed to the Wnt signal, $\beta$-catenin levels are kept low through interactions with the protein kinase GSK-3, APC and Axin. $\beta$-Catenin is degraded, after phosphorylation by GSK-3, through the ubiquitin pathway. (B) Wnt signalling initially leads to a complex between Dsh, Axin and GSK, which may be the regulatory step in the inactivation of GSK. The interaction between Dsh and Axin results in inhibition of GSK that does not phosphorylate $\beta$-catenin anymore, releasing it from the Axin complex with consequent accumulation. The stabilised $\beta$-catenin then enters the nucleus to interact with TCF. This interaction results in an increase in the transcriptional activity.

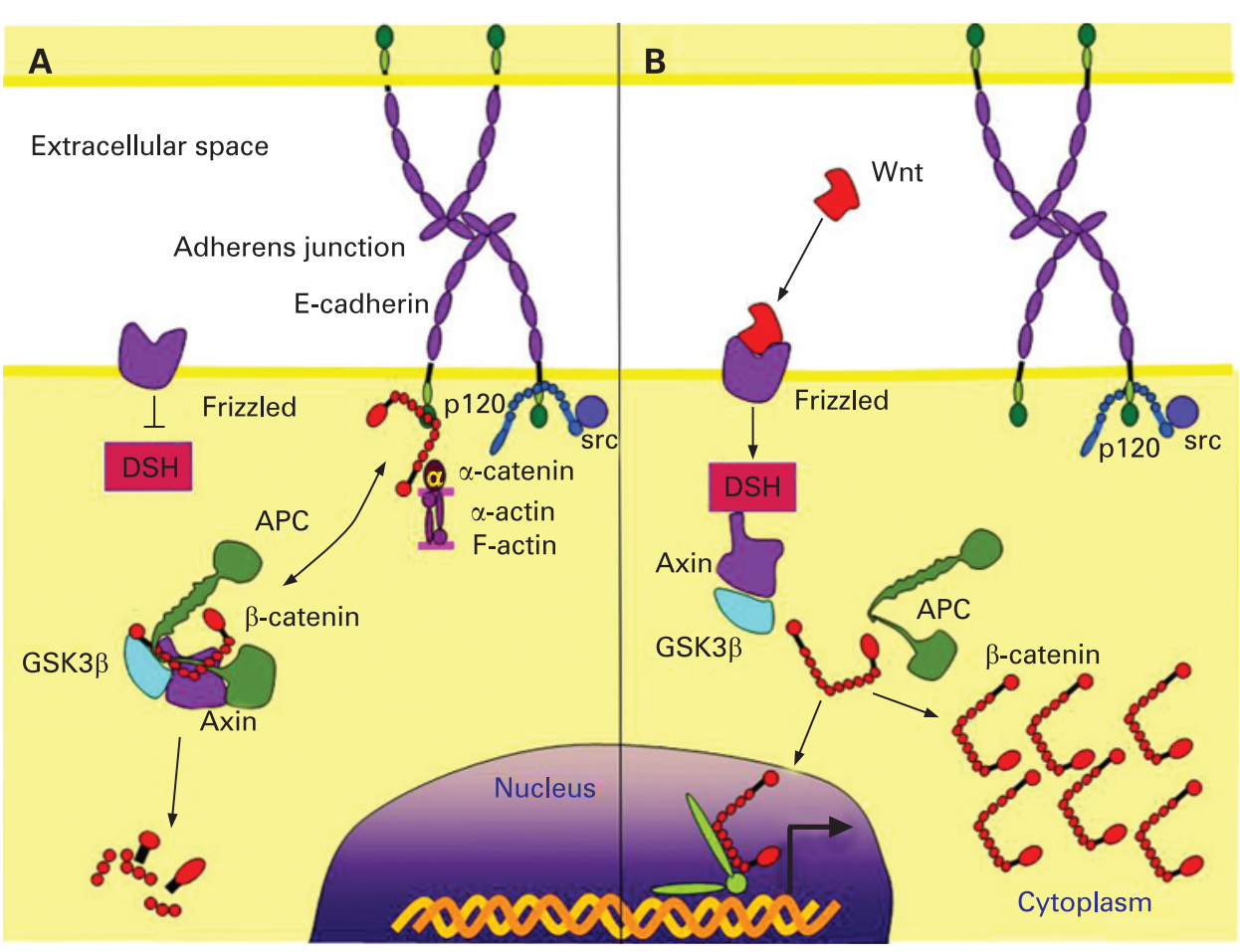




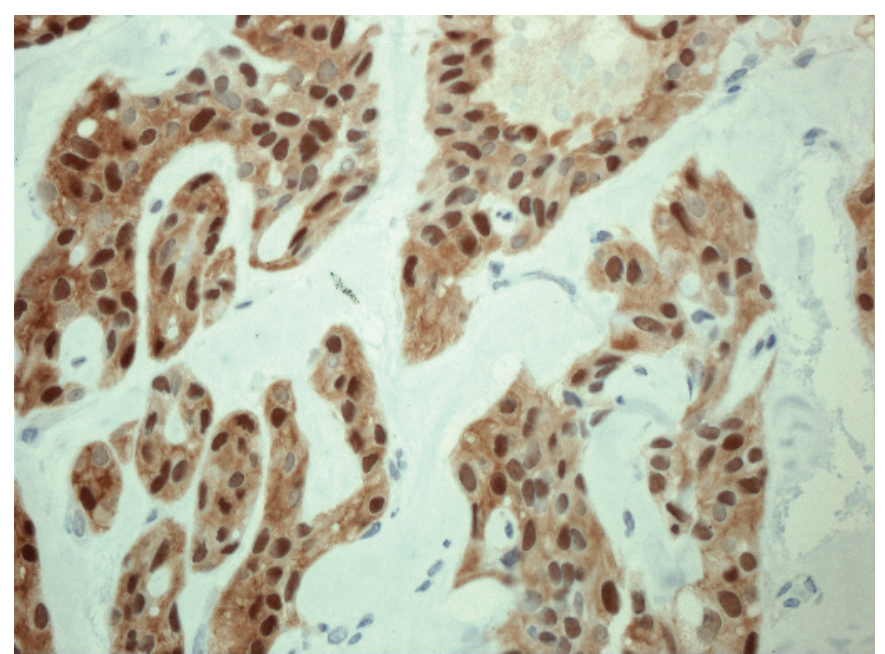

Figure $6 \beta$-Catenin immunostaining yielding characteristic cytoplasmic and nuclear positivity in $100 \%$ of cases of solid pseudopapillary tumour.

CK7 and CK19 are uniformly negative in SPT, and occasional cases are CK8, CK18 and CK20 positive. ${ }^{6}$

It is worth remembering that $\mathrm{AE} 1 / 3$ may also be expressed in occasional metastatic melanomas. ${ }^{12}$

\section{Miscellaneous markers}

Ca19-9 is a marker specific for ductal epithelia but is only focally expressed in a few examples of SPT.

p53 immunoexpression has been encountered in 16\% of cases in a diffuse or patchy distribution. ${ }^{9}$

Vimentin has been reported to be diffusely expressed in SPT with up to $60 \%$ of the tumour population showing positivity. ${ }^{5-7}$ Fifty to $90 \%$ of cases of both melanoma and renal cell carcinomas are also positive for vimentin. ${ }^{10} 13$

\section{Wnt pathway dysfunction}

E-Cadherin and $\beta$-catenin are important molecules in the wnt signalling pathway, and both have been shown recently to be of diagnostic value in SPT. Figure 5 illustrates normal $\beta$-catenin catabolism (A) and interaction with E-cadherin, while (B) is an illustration of the process with wnt pathway activation. The reason these two proteins warrant separation from all the other markers is that they yield consistent results in 100\% of cases of SPT. It is for this reason that they are now regarded as two of the pre-eminent antibodies in the routine panel used to investigate SPT

\section{$\beta$-Catenin}

$\beta$-Catenin is a component of adherens junction that links Ecadherin to $\alpha$-catenin. In the Wnt signalling pathway, $\beta$-catenin plays a key role as a transcriptional activator in conjunction with lymphoid enhancer factor/T cell factor DNA-binding protein to induce target gene expression resulting in cell proliferation and differentiation. ${ }^{14}$ Mutations of the components of the Wnt/ $\beta$-catenin pathway are found in many gastrointestinal cancers. SPT are usually associated with activating mutations in exon 3 of the CTNNB1 gene encoding for $\beta$-catenin in $83 \%$ of cases, resulting in dysregulation and redistribution of $\beta$-catenin protein leading to characteristic strong nuclear and cytoplasmic immunoreactivity in $100 \%$ of the cases (fig 6). ${ }^{14-16}$ This staining pattern contrasts with the normal pancreas, where $\beta$-catenin protein shows distinct membrane decoration of the ducts and the acini. The rationale for this aberrant location is because the mutations involve GSK$3 \beta$ phosphorylation sites and abrogate subsequent ubiquitinmediated degradation of $\beta$-catenin protein, allowing for cytosolic accumulation and pooling of $\beta$-catenin with consequent nuclear shift leading to nuclear accumulation (see fig 7 , area $\mathrm{A}$ )

While activation of the Wnt pathway results in relocation of $\beta$-catenin protein, it also results in overexpression of Cyclin D1 and glutamine synthetase. ${ }^{16}$ Cyclin D1 is variably overexpressed
Figure 7 (A) Activating mutations in the human $\beta$-catenin gene found in human cancers. These mutations alter specific $\beta$ catenin residues important for GSK3 phosphorylation (white circles represent the mutated phosphorylation sites) and stability, resulting in inhibition of $\beta$ catenin degradation, loss of membranous localisation with cytoplasmic and nuclear accumulation and interaction with $T$ cell factor. (B) Schematic representation of the presenilin-1 (PS1) $/ \gamma$-secretasemediated disassembly of cadherins of the adherens junction. Matrix metalloproteinase (MMP)-mediated proteolytic activity cleaves the extracellular domain of cytoskeletal Ecadherin and releases it to the extracellular medium (1). A fragment of Ecadherin containing the transmembrane and cytoplasmic sequence of E-cadherin remains bound to PS1. This fragment is then cleaved by a PS1/ $\gamma$-secretase activity at the membrane-cytosol interface to produce a fragment of $\beta$ catenin that dissociates from both PS1 and is released into the cytosol (2) and then enters the nucleus (3).

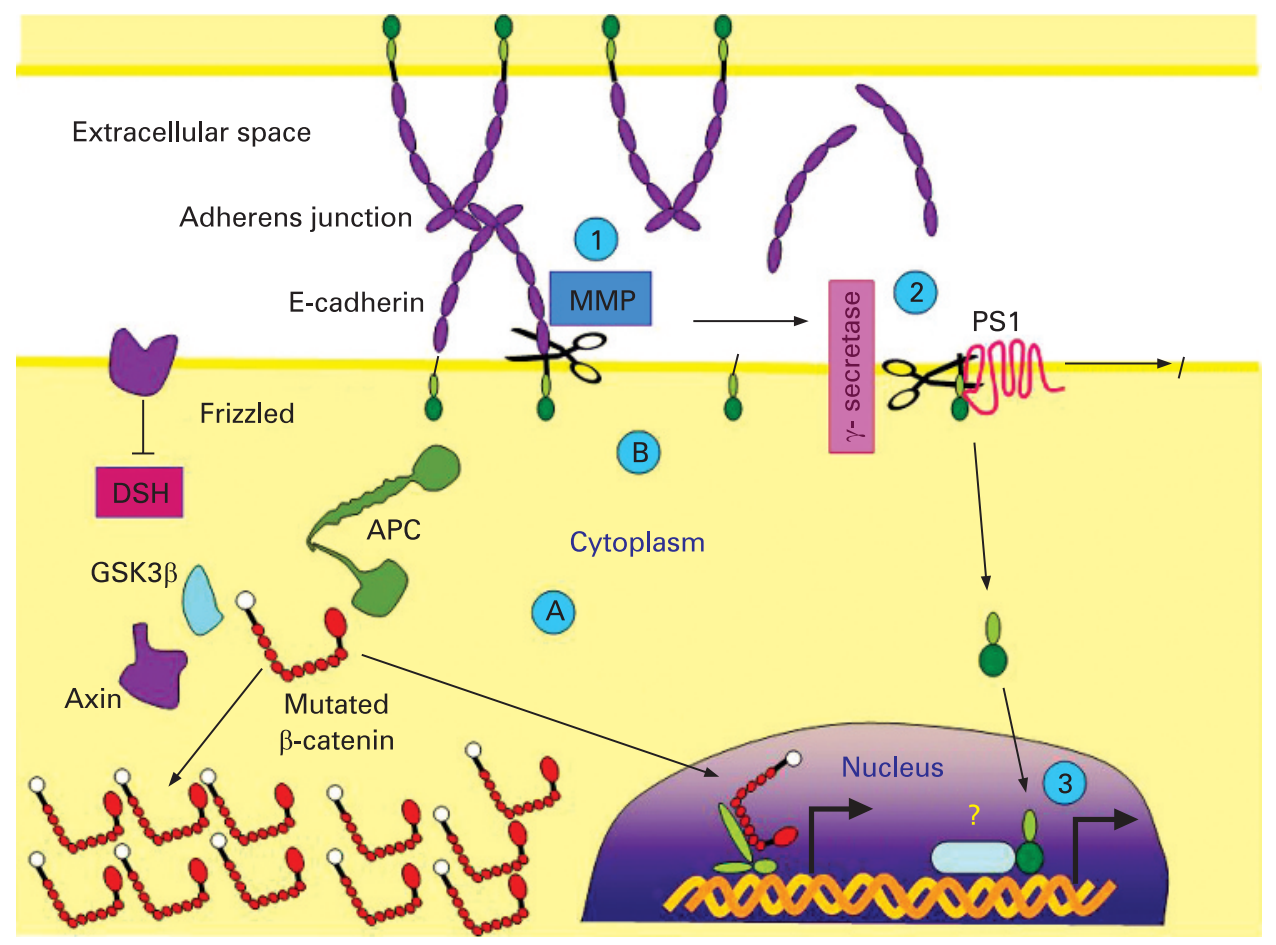


E-cadherin

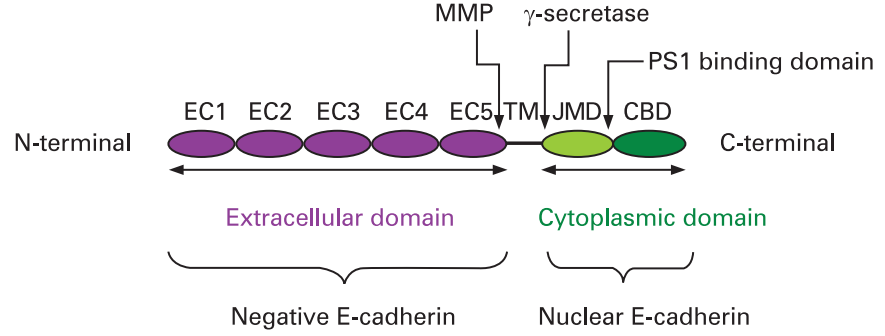

Figure 8 Schematic representation of E-Cadherin protein, with the extracellular domain (EC), Juxtamembrane Domain (JMD) and Catenin Binding Domain (CBD), together with cleavage sites for matrix metalloproteinase (MMP) and $\gamma$-secretase, and the binding domain for presenilin-1 (PS1). Antibodies against E-cadherin that recognize the extracellular domain result in an absence of staining, while antibodies against the cytoplasmic domain result in a nuclear staining.

in SPTs from $74 \%$ to $100 \%$ of the cases and variably from $15 \%$ to $100 \%$ of the tumour cells. ${ }^{14}{ }^{16}$ These antibodies are negative in the surrounding normal pancreas. Glutamine synthetase shows strong cytoplasmic immunostaining in SPT, in contrast to the weak cytoplasmic staining observed in the surrounding normal exocrine and endocrine pancreas. ${ }^{16}$

Thus, there is a strong correlation between mutations in the $\beta$-catenin gene and the resultant aberrant protein expression in SPT. While the vast majority (85-90\%) of SPT show exon 3 mutations, $100 \%$ of cases show nuclear and cytoplasmic immunoreactivity. In those cases not showing exon 3 mutations, it is likely that mutations are present in other exons that would account for the remaining $10-15 \%$ of cases.

Nuclear expression of $\beta$-catenin is extremely uncommon in pancreatic neuroendocrine tumours. ${ }^{17}$

\section{E-Cadherin}

E-Cadherin is a calcium-dependent transmembrane glycoprotein, localised to zonula adherens junctions at the basolateral surface in epithelial cells and is involved in cell-cell interaction. Normal E-cadherin expression plays a key role in the maintenance of epithelial integrity and polarity function. The Ecadherin molecule is composed of a cytoplasmic domain, a single-pass transmembrane domain and an extracellular domain that consists of five tandemly repeated cadherin-motifs subdomains with putative calcium-binding sites. The cytoplasmic domain of E-cadherin interacts with the catenin molecules that mediate its binding to the actin cytoskeleton (see fig 8).

E-cadherin binds to either $\beta$-catenin or $\gamma$-catenin. E-cadherin immunostaining is localised to and highlights the cell membrane of normal cells of pancreatic ducts and acini as well as endocrine cells.

There has been a recent flurry of publications on E-cadherin expression in SPT. ${ }^{16}$ 18-21 In addition to some observing loss of membrane expression, others have also noticed very characteristic nuclear positivity for E-cadherin. ${ }^{721}$ Nuclear E-cadherin expression was explored further in another set of tumours (oesophageal squamous carcinomas), and in fractionation studies, a fragment of the E-cadherin molecule was confirmed to be intranuclear in location. ${ }^{22}$ Further to this, our group showed that the staining pattern for E-cadherin in SPT is dependent on the type of E-cadherin antibody used. ${ }^{19}$ Use of the antibody that recognises the extracellular domain of the Ecadherin molecule results in an absence of staining in SPT (complete loss membrane staining in all cases) (fig 9A,B), while
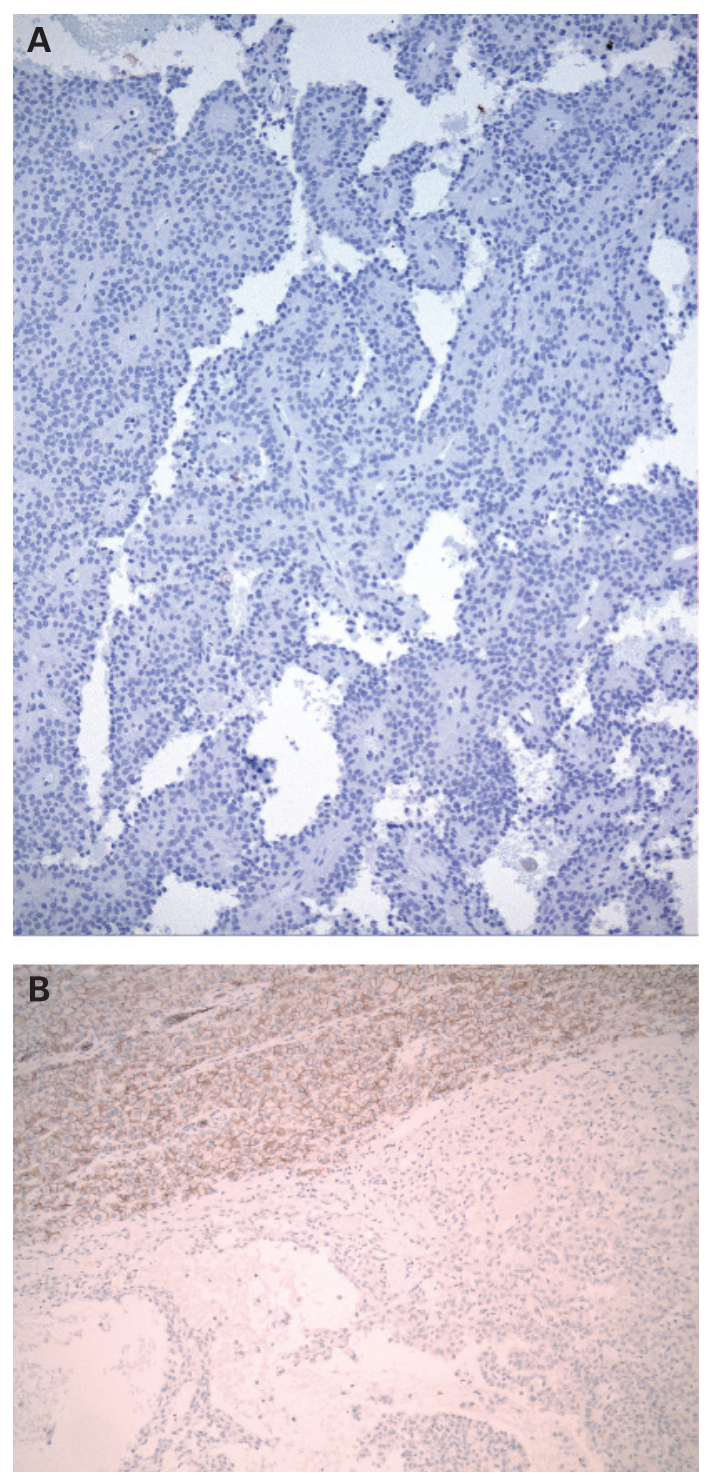

Figure 9 Loss of membrane staining for E-cadherin in the primary solid pseudopapillary tumour (SPT) (A) when using the antibody that recognises the extracellular domain of the molecule. This is seen in $100 \%$ of cases of SPT. This loss of membrane staining is also retained in metastatic lesions where normal hepatocytes (top) show membrane staining for E-cadherin, while the metastatic SPT is completely negative (B).

use of the antibody that recognises the cytoplasmic domain results in nuclear positivity (fig 10). ${ }^{19}$

Nuclear immunoreactivity of E-cadherin with loss of membranous staining was found in all cases studied, using an antibody that recognises the intracytoplasmic domain of Ecadherin. ${ }^{19}{ }^{21}$ Cytoplasmic immunoreactivity with variable dotlike pattern ${ }^{7} 16$ or a complete loss of membrane staining and absence of cytoplasmic immunoreactivity was seen using an antibody against the extracellular domain of E-cadherin. ${ }^{19} 20$ The significance of dot-like E-cadherin cytoplasmic positivity in SPT is not known; however, cases of gastric cancer with some types of CDH1 gene mutations have led to Golgi accentuation of Ecadherin immunohistochemistry.

Approximately $30 \%$ of pancreatic neuroendocrine tumours have also been documented as showing nuclear immunoreactivity for E-cadherin using the antibody to the intracytoplasmic domain and loss of membrane staining with the antibody to the 


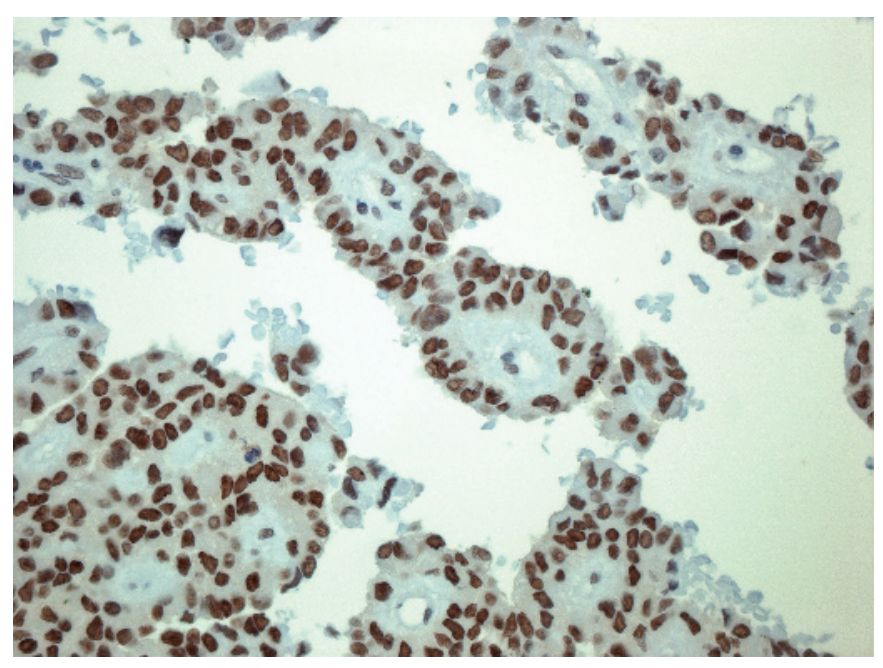

Figure 10 Application of the E-cadherin antibody raised against the cytoplasmic fragment yielding strong nuclear staining. There is usually complete concordance when both E-cadherin antibodies are used.

extracellular domain. ${ }^{16}$ Thus, nuclear E-cadherin positivity is not the exclusive preserve of SPT and can be seen in pancreatic neuroendocrine tumours, Merkel cell carcinomas and approximately $50 \%$ of clear cell renal cell carcinomas. ${ }^{23}{ }^{24}$

Thus, it is important to be aware of the E-cadherin antibody that is employed in the work-up of SPT, as different immunohistochemical results will be obtained.

The rationale for the consistent E-cadherin aberrations is not clear cut. It has been suggested that p120, an E-cadherin regulator, rather than mutations of the $\mathrm{CDH} 1$ gene, may be responsible for dysregulation of E-cadherin protein in SPT. ${ }^{25}$

E-cadherin immunohistochemistry is of diagnostic value in all cases of SPT as all cases will show either nuclear positivity or absence of membrane staining depending on the antibody that is employed.

\section{CONCLUSION}

Table 1 summarises the immunohistochemical findings of SPT and its lookalikes.

As can be seen from the discussion, there have been several markers that have been employed in the immunohistochemical work-up of SPT. This brief overview is not intended to be all encompassing but rather to highlight the important antibodies

\section{Take-home points}

Solid pseudopapillary tumours (SPT) may show histological overlap with other tumours such as endocrine tumours.

- The key immunohistochemical markers in the evaluation of SPT are: CD10, progesterone receptor, synaptophysin, $\beta$ catenin and E-cadherin.

- $\beta$-Catenin staining of $100 \%$ of SPT is cytoplasmic and nuclear, and is reflective of mutations in the gene.

- E-Cadherin immunostaining is dependent on the type of antibody used: nuclear staining is seen with the antibody to cytoplasmic domain, while complete loss of membrane localisation is encountered with the antibody to the extracellular domain.

- The immunoprofile of CD10 +ve, PR +ve, $\beta$-catenin cytoplasmic and nuclear +ve, E-cadherin nuclear +ve/ membrane loss is virtually diagnostic of SPT.

and their immunoreactivity with SPT. It is worth reiterating that most SPT are readily recognised morphologically, but predominantly solid variants, those with a significant clear cell component and needle biopsy material, can all be quite challenging. In the case of metastatic renal cell carcinoma or malignant melanoma, an appropriate clinical history is ideal; however, there are times when this information is not forthcoming. With regards to morphologically diagnostic cases, it is a moot point whether any immunohistochemistry needs to be performed at all. It is perhaps advisable to perform confirmatory immunohistochemistry if these tumours are not encountered frequently, even though the diagnosis of SPT may be obvious. As with most tumours, a panel of markers is advocated, and the results thereof need to be correlated with morphology and clinicoradiological information. It is important to remember that one antibody or marker alone cannot identify SPT; hence a prudent panel of markers is advocated. Although further analysis of the expression of different members of the wnt pathway in renal cell carcinoma is still required, a tumour that is CD10 positive, displays cytoplasmic and nuclear $\beta$-catenin immunolabelling, and shows nuclear E-cadherin expression with the antibody to the cytoplasmic domain and loss of membrane staining with the antibody to the extracellular domain is likely to be a SPT.

Competing interests: None.

Table 1 Common immunohistochemical panel of antibodies for solid pseudopapillary tumour (SPT) and its mimics

\begin{tabular}{|c|c|c|c|c|c|}
\hline Antibody & SPT & PET & ACC & RCC & MM \\
\hline CD56 & Focally positive in up to $100 \%$ & Positive in $25-50 \%$ & Positive & Positive & Positive \\
\hline CD10 & Positive in $80 \%$ & Positive in up to $10 \%$ & Positive & Positive & Positive \\
\hline PR & Positive & Positive & Positive & Negative & Positive \\
\hline Synaptophysin & Positive in up to $70 \%$ & Positive $100 \%$ & Positive & Negative & Negative \\
\hline$\beta$-Catenin & Nuclear/cytopositivity $100 \%$ & Nuclear/cytopositivity in rare cases & $?$ & $?$ & $?$ \\
\hline \multirow[t]{2}{*}{ E-cadherin } & Nuclear* $100 \%$ & Nuclear* $30 \%$ & $?$ & $\begin{array}{l}\text { Nuclear* } 50 \%- \\
\text { clear cell variant } \\
\text { only }\end{array}$ & Nuclear not described \\
\hline & Loss of membrane staining $100 \%$ & $\begin{array}{l}\text { Retention of membrane staining } 95- \\
100 \%\end{array}$ & & $\begin{array}{l}\text { Retention of } \\
\text { membrane staining } \\
\text { in majority }\end{array}$ & $\begin{array}{l}\text { Occasional membrane } \\
\text { loss }\end{array}$ \\
\hline
\end{tabular}

*Nuclear immunoreactivity with the antibody to the cytoplasmic domain of E-cadherin.

ACC, acinar cell carcinoma; cyto, cytoplasmic; MM, malignant melanoma; PET, pancreatic endocrine tumour; RCC, renal cell carcinoma. 


\section{REFERENCES}

1. Edirimanne S, Connor SJ. Incidental pancreatic cystic lesions. World J Surg 2008:32:2028-37.

2. Campbell F, Azadeh B. Cystic neoplasms of the exocrine pancreas. Histopathology 2008:52:539-51.

3. Santini D, Poli F, Lega S. Solid-papillary tumors of the pancreas: histopathology. J Pancreas 2006; 7:131-6.

4. Saiura A, Umekita N, Matsui Y, et al. Successful surgical resection of solid cystic tumor of the pancreas with multiple liver metastases and a tumor thrombus in the portal vein. Hepatogastroenterol 2000;47:887-9.

5. Albores-Saavedra J, Simpson KW, Bilello SJ. The clear cell variant of solid pseudopapillary tumor of the pancreas: a previously unrecognized pancreatic neoplasm. Am J Surg Pathol 2006;30:1237-42.

6. Notohara K, Hamazaki S, Tsukayama C, et al. Solid pseudopapillary tumor of the pancreas: immunohistochemical localization of neuroendocrine markers and CD10. Am J Surg Pathol 2000;24:1361-71.

7. Serra S, Salahshor S, Fagih M, et al. Nuclear expression of E cadherin in solid pseudopapillary tumors of the pancreas. J Pancreas 2007;8:296-303.

8. Adams AL, Siegal GP, Jhala NC. Solid pseudopapillary tumor of the pancreas. Adv Anat Pathol 2008:15:39-45.

9. Viale G, Doglioni C, Gambacorta M, et al. Progesterone receptor immunoreactivity in pancreatic endocrine tumors. An immunocytochemical study of 156 neuroendocrine tumors of the pancreas, gastrointestinal and respiratory tracts, and skin. Cancer 1992; 70:2268-77.

10. Fitzgibbons $\mathbf{P L}$, Chaurushiya PS, Nichols PW, et al. Primary mucosal malignant melanoma: an immunohistochemical study of 12 cases with comparison to cutaneous and metastatic melanomas. Hum Pathol 1989;20:269-72.

11. Rasmuson T, Grankvist K, Roos G, et al. Neuroendocrine differentiation in renal cell carcinoma- evaluation of chromogranin and neuron-specific enolase. Acta Oncol 1999;38:623-8.

12. Plaza JA, Suster D, Perez-Montiel D. Expression of immunohistochemical markers in primary and metastatic malignant melanoma: a comparative study in 70 patients using a tissue microarray technique. Appl Immunohistochem Mol Morphol 2007:4:421-5.

13. Kim MK, Kim S. Immunohistochemical profile of common epithelial neoplasms arising in the kidney. Appl Immunohistochem Mol Morphol 2002;10:332-8.
14. Abraham SC, Klimstra DS, Wilentz RE, et al. Solid-pseudopapillary tumors of the pancreas are genetically distinct from pancreatic ductal adenocarcinomas and almost always harbor beta-catenin mutations. Am J Pathol 2002;160:1361-9.

15. Tanaka Y, Kato K, Notohara K, et al. Frequent beta-catenin mutation and cytoplasmic/nuclear accumulation in pancreatic solid-pseudopapillary neoplasm. Cancer Res 2001;61:8401-4.

16. Audard V, Cavard C, Richa $\mathrm{H}$, et al. Impaired E-cadherin expression and glutamine synthetase overexpression in solid pseudopapillary neoplasm of the pancreas. Pancreas 2008:36:80-3

17. Chetty R, Serra S, Asa SL. Loss of membrane localization and aberrant nuclear Ecadherin expression correlates with invasion in pancreatic endocrine tumors. Am J Surg Pathol 2008;32:413-19.

18. Tang WT, Stelter AA, French $S$, et al. Loss of cell-adhesion molecule complexes in solid pseudopapillary tumor of the pancreas. Mod Pathol 2007;20:509-13.

19. Chetty R, Serra S. Membrane loss and aberrant nuclear localization of E-cadherin are consistent features of solid pseudopapillary tumour of the pancreas. An immunohistochemical study using two antibodies recognizing different domains of the E-cadherin molecule. Histopathology 2008;52:325-30.

20. Kim MJ, Jang SJ, Yu E. Loss of E-cadherin and cytoplasmic-nuclear expression of beta-catenin are the most useful immunoprofiles in the diagnosis of solidpseudopapillary neoplasm of the pancreas. Hum Pathol 2008;39:251-8.

21. El-Bahrawy MA, Rowan A, Horncastle D, et al. E-cadherin/catenin complex status in solid pseudopapillary tumor of the pancreas. Am J Surg Pathol 2008;32:1-7.

22. Salahshor S, Naidoo R, Serra S, et al. Frequent accumulation of nuclear E-cadherin and alterations in the wnt signaling pathway in esophageal squamous cell carcinomas. Mod Pathol 2008;21:271-81.

23. Han AC, Soler AP, Tang CK, et al. Nuclear localization of E-cadherin expression in Merkel cell carcinoma. Arch Pathol Lab Med 2000;124:1147-51.

24. Gervais ML, Henry PC, Saravanan A, et al. Nuclear E-cadherin and VHL immunoreactivity are prognostic indicators of clear-cell renal cell carcinoma. Lab Invest 2007;87:1252-64.

25. Chetty R, Jain D, Serra S. p120 catenin (p120) reduction and cytoplasmic relocalization leads to dysregulation of E-cadherin in solid pseudopapillary tumors of the pancreas. Am J Clin Pathol 2008;130:71-6.

\section{Drug and Therapeutics Bulletin (DTB)}

Your key source of unbiased, independent advice

For over 45 years DTB has been an independent, indispensable part of evidence-based clinical practice. DTB offers healthcare professionals detailed assessment of, and practical advice on, individual medicines and other treatments, groups of treatment and the overall management of disease.

DTB is now also available online at http://dtb.bmj.com:

- browse or search all DTB content from the latest issue back to 1994

- email alerting, sophisticated searching, RSS feeds and full text links from cited references

- interactive services such as My Folders for quick access to articles that you have viewed previously and My Searches to save and re-use useful searches

- comment online on any DTB article

To subscribe, or for further information, please visit http://dtb.bmj.com 